\title{
Detection of HSV1 and HSV2 viruses among Iraqi patients with Recurrent Stomatitis Aphthous
}

\author{
Eman Abid Fahad Al-Hasnawi \\ Msc. Dentistry science /oral Microbiology - Medical Technical Institute/Al-Mansour - Middle Technical University
}

\begin{abstract}
\end{abstract}
Background: Recurrent aphthous stomatitis (RAS) is a global oral case that stay incompletely understood yet. It was classified into three main types of minor, major or herpetiform, based on clinical features, but the exact causative agent is unknown, therefore treatments are primarily should be experimental and aimed at decrease symptoms rather than remedy or prevention. The aim of study is to detection of Herpes simplex virus-1 and Herpes simplex virus-2 in saliva of patients with recurrent aphthous stomatitis (RAS) as a causative agents of the disease by the enzyme-linked immunosorbent assay (ELISA) assay, and correlation between positive results and finding of age, gender, and family history.

Materials and Methods: Study group of (40) saliva samples were taken from 40 patients with RAS lesions included 15 male and 25 female, with mean age at (34) years, at range about (18-55) years, and healthy control group were (20) healthy volunteers were suffered from oral traumatic ulcers, consist women (13), men (7), mean age (33) years in range (18-55). for detection of HSV viruses types that may causes this disease, and healthy control group were (20) healthy volunteers were suffered from oral traumatic ulcers, ELISA method used for diagnosis HSV-1 and HSV-2, from saliva samples of the patients with (RAS) with correlation between positive results and finding of age, gender, and family history.

Results: Diagnostic specimens were consisted of two groups, study group of (40) saliva samples taken from 40 patients with RAS lesions. The results of ELISA to detect HSV had high significant (17): 42.5\% from (40) patients with (RAS), HSV-1: (13)32.5\%, and HSV-2: (4) $10 \%$.

Conclusion: This high number of RAS cases by HSV, and presence of HSV-2 in patients saliva in this study acted important cause of prevalence of this disease in Iraq, were in age group (18-25) mostly in female especially in those with family history of infection.

\section{KEYWORDS}

Recurrent Aphthous Stomatitis (RAS), Aphthous ulcer, HSV-1\&2 viruses, ELISA method.

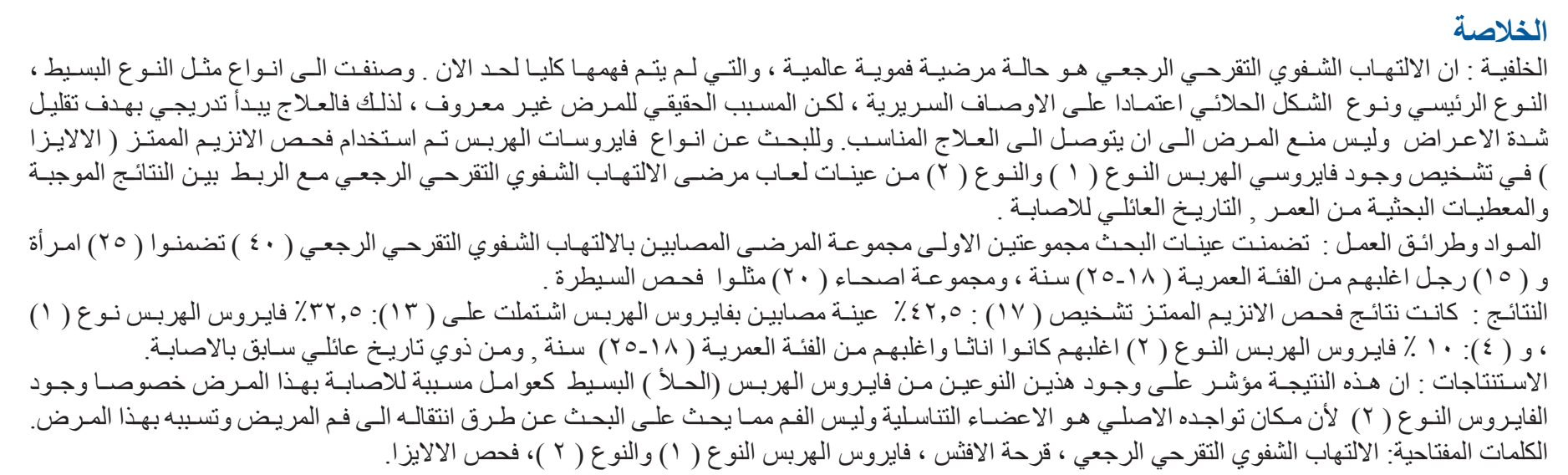

\section{INTRODUCTION}

Recurrent aphthous stomatitis (RAS) is a painful disease of the oral mucosa that affects $20 \%$ of the general population worldwide population. It is one of the most common oral complaints that may seen in the primary care setting. Despite its still prevalence, its causes remain largely unknown yet ${ }^{(1)}$.

"Aphtae (Greek aphtai, means burn) are ulcerated lesions that sit on the mucosal surface where, unlike erosion, loss of continuity involves the whole epithelial lining and may affect the underlying connective tissue" (2).

The cause is still unknown, therefore the diagnosis is also usually based on medical history and symptomatic findings and the laboratory procedures cannot exist in the surely diagnosis ${ }^{(3)}$.

Many factors are known to contribution to the presence of oral aphthae, that is including hereditary and some genetic factors, local trauma, food allergens, hormones changing ranges (estrogen and progesterone), anxiety, stress, smoking, some of chemical materials and microbiological materials (4). There are many systemic disorders causes that known to be have association with oral aphthae, such as the case of (Behçet's syndrome), some vitamins deficiencies, hematological disorders, acute gastrointestinal infections, Reiter syndrome, neutropenia, (Magic syndrome), (pharyngitis and cervical adenopathy aphthous), and immune deficiencies ${ }^{(5)}$. There are three clinical types of RAS established according to the severity, number and the duration of outbreaks ${ }^{(6)}$.

- Minor (RAS): This is the most common presentation of the disease, representing $70-85 \%$ of all cases. It manifests as small rounded lesions, has a grayish-white color and encircled by an erythematous 
ring. Each minor RAS digression usually includes the presence of (1-5) ulcers of (1) $\mathrm{cm}$ in diameter. These ulcers are auto limiting and recover within (4-14) days without causing scars ${ }^{(7)}$.

- Major (RAS): This is the most severe appearance of the disease, representing as (10\%) of all cases. In this subtype the size of ulcers is over (1) $\mathrm{cm}$, and tend to infect the lips, pharynx and even soft palate. The lesions remain for up to (6) weeks and then leave scars ${ }^{(8)}$.

- Herpetiform (RAS): This type may reach to (1-10\%) of all diagnosed cases and is recognized by occurring of recurrently have been contagious of deep, divers small, and usually painful ulcers. This finding is usually seen mostly in women and also in patients with older age, while the other two types of this disease haven't these properties ${ }^{(4)}$.

The real diagnosis of RAS is often based on the medical history of patient and also on the clinical exhibitions. There is no specific and confirmed diagnostic test yet, also it is essential to dismiss the possible fundamental systemic causes especially in those adults who suffer abrupt RAS outbreaks ${ }^{(9)}$. Many recent studies get documented a correlation between RAS and a many psychological factors are effect on infection including repressed hostility, anxiety, as well as nature of job related and many type of stressors (10). "A subset of patients with RAS are predisposed to develop aphthae at sites of trauma", local trauma such as toothbrushing, anaesthetic injections, and dental treatment would induces aphthous ulceration to occur in these patients, and this developing is still unknown (11). Several reports document the negative association between smoking and the occurrence of (RAS). There is only several positive cases association has been documented with use of less smoke tobacco (chewing tobacco and also snuff tobacco) ${ }^{(12)}$. Bacterial agents In 1963 that isolated were Streptococccus oralis from many aphthous ulcerated lesion. Other studies by Donatsky 1975, found high titers of many antibodies were effected certain oral bacteria, such as strains of streptococci in some patients with RAS when he compared them with controls. antibacterial antibodies Cross-reaction test of contributed with oral mucosa then contended as an inducing agents of immune pathogenic mechanism in RAS ${ }^{(13)}$. Later studies, have not confirmed all of these findings. In a serological tests, founding that Helicobacter pylori has not always presence as a significant causative factor in all the appearance of RAS. Also some of studies had diagnosed H. pylori (DNA) in several samples from many lesions contributed to RAS by using Polymerase chain reaction $(\mathrm{PCR})$ assay ${ }^{(14)}$.

A certain viral causes of RAS has been detected by several researchers. Sallay et al in (1973) said: "isolated adenoviruses from oral aphthae, but there was no antibody response to adenovirus in RAS. Adenoviruses are usually ubiquitous organisms and these results need confirmation"(15). Many recent studies detected HSV-1 DNA in such a several samples of biopsies from oral ulcers of aphthae from many cases of RAS patients. Some of studies were unable to detect any HSV antigen in the biopsies samples, and even HSV cannot be able to isolated from RAS lesions culture ${ }^{(16)}$.

Antivirals drugs like acyclovir, are very effective against HSV, present to have only equivocal clinical effect on RAS lesions. Further studies have also detected Varicella zoster virus-like DNA ${ }^{(17)}$.

\section{PATIENTS AND METHODS}

Sample description: There were 40 individuals included in this study design . They were seen in many dentistry clinics in Baghdad at period from April 2016 till January 2017.

Questionnaire: Age \& gender, oral hygiene, family history of disease with medical History, job, age, period of disease, presence of systemic disease.

Saliva collection: Five ml of whole un stimulated saliva were collected from all the participants using a sterile plastic polyethylene bottles. The saliva then centrifuged at $3000 \mathrm{rpm}$ for 10 minutes, this was done within (1) hour after collection to excrete any debris and cellular substances, and the supernatants were suck out immediately into two labeled cuvettes tubes and stored frozen at $\left(-20^{\circ} \mathrm{C}\right)$ until assayed.

ELISA Method: Patients or diagnostic specimens were consisted of two groups. The first group was comprised of (40) saliva samples with RAS lesions were taken from 40 patients included (25) women and (15) men, with mean age (34) years, range (18$55)$, with had RAS lesions of the minor and herpetic types. The second group samples were taken from 20 healthy volunteers suffered from oral traumatic ulcers as a control (women were (13), men were (7), with mean age (33) years, at range about (18-55) with no history of any oral lesions or disease. This study was carried out from April in 2016 to January in 2017 and the samples in this study were collected from many clinics of Dentistry in many regions in Baghdad and conducted in many laboratories, ELISA Method was done for detection of herpes viruses (HSV-1) and (HSV-2) by using Human HSV-1 and HSV-2 IgG enzyme immunoassay kits in saliva samples, US 
Biological HN-1 glycoprotein C for enzyme linked Immunoassay kit for detection of HSV-1\& HSV-2 viral $\mathrm{Ag}^{(18)}$.

Statistics: Used Microsoft Excel 2010 (Field EA, 2012) a. Tables, b. Numbers (No.), c. Percentage $(\%)$, d. Mean e. Bar charts.

\section{RESULTS}

\section{1- Patients with RAS lesions:}

Table (1):

In this table, saliva samples with RAS lesions taken from 40 patients included (25) women and (15) men, mean age were (34) years, from age range (18-55), study group had RAS lesions of the minor type and herpetic type. The control were (20) healthy volunteers suffered from oral traumatic ulcers were women (13), men (7), mean age (33) years in range (18-55).

Table (1): Description of Study group with Age, Gender, Family history and Control

\begin{tabular}{c|c|c|c|c|c|c|c|c|c|c|c|}
$\begin{array}{c}\text { Age groups } \\
\text { Mean age }\end{array}$ & $\begin{array}{c}\boldsymbol{R} \text { AS } \\
\text { (34) }\end{array}$ & \multicolumn{4}{|c|}{ Gender } & \multicolumn{4}{c|}{ Family history } & $\begin{array}{c}\text { Healthy control } \\
\text { N= 20 }\end{array}$ \\
\hline$(18-25)$ & 23 & 9 & 22.5 & 14 & 35 & 11 & 27.5 & 12 & 30 & 9 \\
\hline$(26-35)$ & 11 & 4 & 10 & 7 & 17.5 & 4 & 7.5 & 8 & 20 & 6 \\
\hline$(36-45)$ & 4 & 1 & 2.5 & 3 & 7.5 & 2 & 2.5 & 2 & 5 & 2 \\
\hline$(46-55)$ & 2 & 1 & 2.5 & 1 & 2.5 & 0 & 0 & 1 & 2.5 & 3 \\
\hline Total & 40 & 15 & 37.5 & 25 & 62.5 & 17 & 42.5 & 23 & 57.5 & 20 \\
\hline
\end{tabular}

(46-55)

$(36-45)$

(26-35)

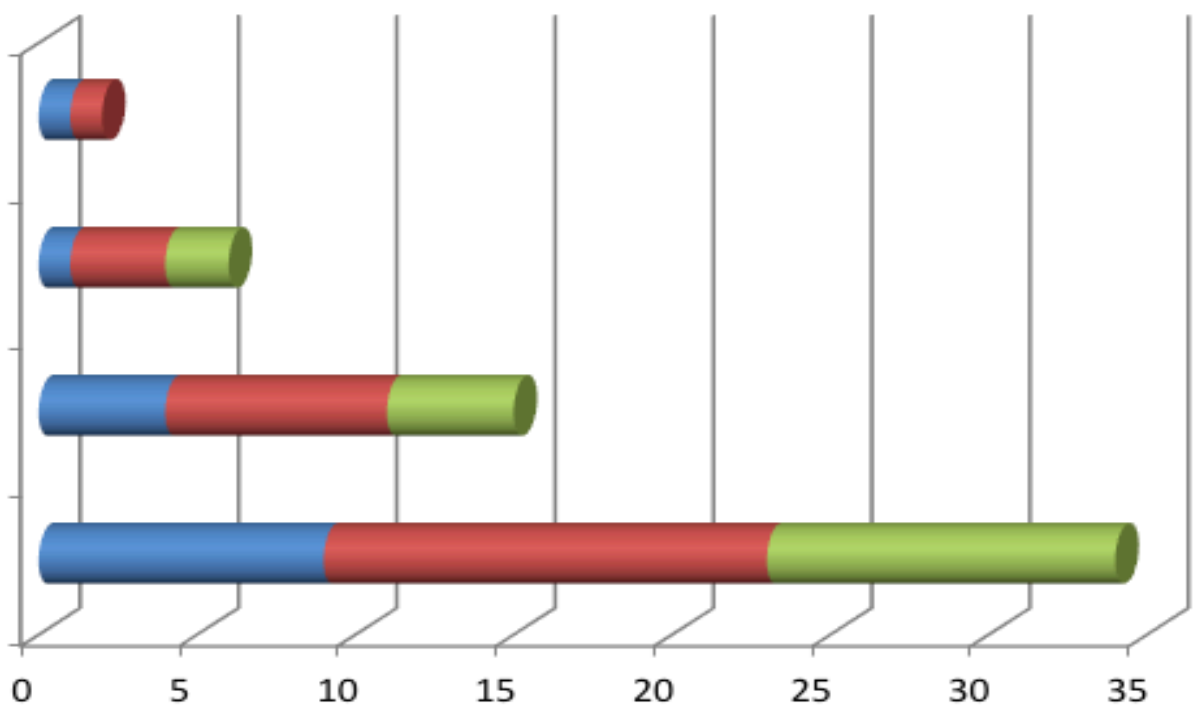

Male

Female

Family history

Figure (1): Description of study group with Age, Gender, Family history

\section{2- ELISA Results of HSV:}

Table (2):

This table showed ELISA results that appeared

(17) positive cases with HSV, the most were in age

Table (2): ELISA Results of HSV in Study group with Gender and Healthy group

\begin{tabular}{|c|c|c|c|c|c|c|c|c|c|}
\hline Age groups & $R A S \quad N=40$ & \multicolumn{4}{|c|}{ ELISA test of HSV } & \multicolumn{2}{|c|}{ Gender } & \multicolumn{2}{|c|}{$\begin{array}{c}\text { Healthy control } \\
\qquad \begin{array}{c}N=40\end{array}\end{array}$} \\
\hline & & $+v e$ & $\%$ & $--v e$ & $\%$ & $M$ & $F$ & $+v e$ & $--v e$ \\
\hline$(18-25)$ & 23 & 8 & 20 & 15 & 37.5 & 3 & 5 & 2 & 7 \\
\hline$(26-35)$ & 11 & 6 & 15 & 5 & 12.5 & 2 & 4 & 1 & 5 \\
\hline$(36-45)$ & 4 & 2 & 5 & 2 & 5 & 0 & 2 & 0 & 2 \\
\hline$(46-55)$ & 2 & 1 & 2.5 & 1 & 2.5 & 1 & 0 & 0 & 3 \\
\hline Total & 40 & 17 & 42.5 & 23 & 57.5 & 6 & 11 & 3 & 17 \\
\hline
\end{tabular}




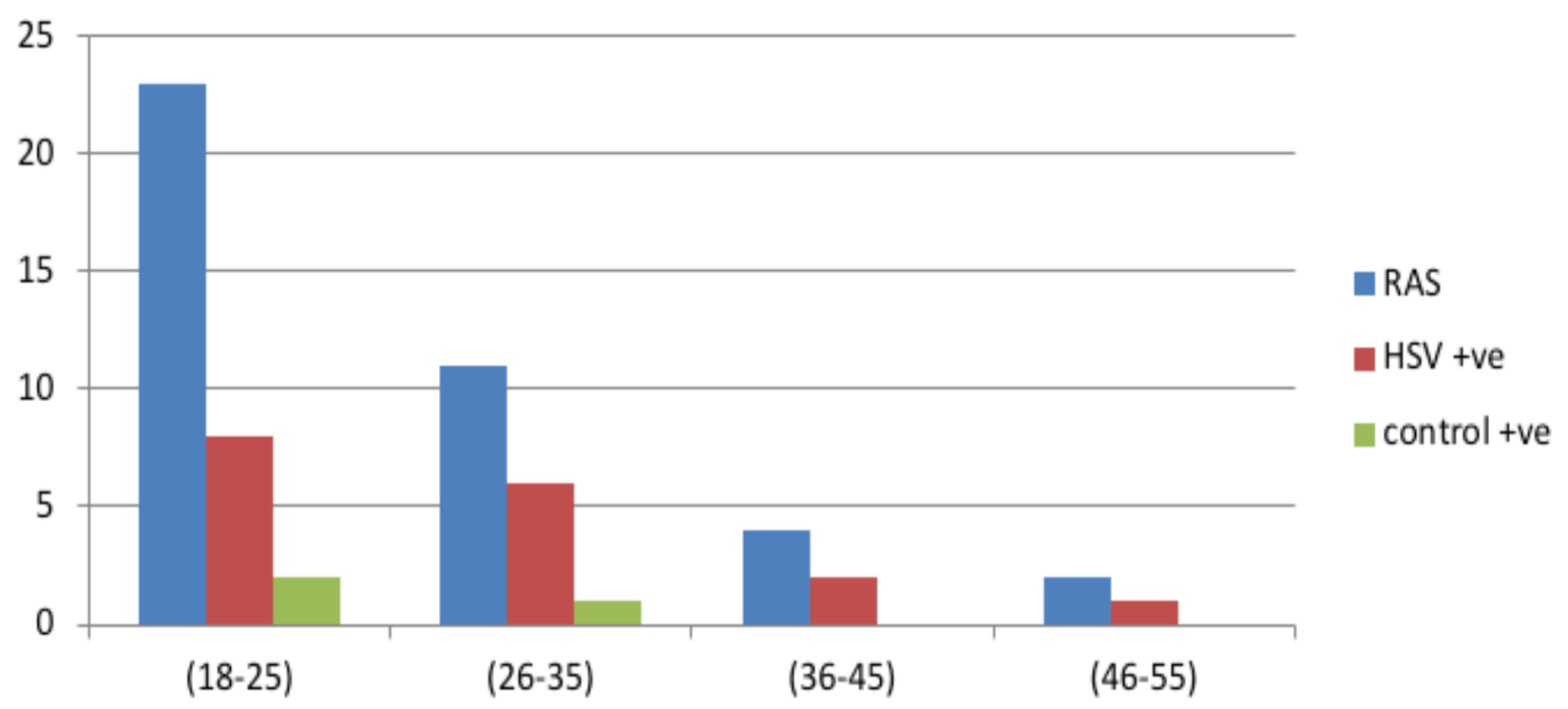

Figure (2): ELISA test of HSV results in Study groups with Aphthus and Control group

\section{3- Results of HSV-1 and HSV-2:}

was in total of (4) most of them in age group (26-35)

Table (3): Showed ELISA results of HSV in while in health control group was slightly presence study age groups with aphthus by HSV-1 was in total mostly in age group (18-25).

of (13) most of them in age group (18-25) and HSV-2

Table (3): ELISA results of HSV in Study group Age groups with Aphthus by HSV-(1) and HSV-(2)

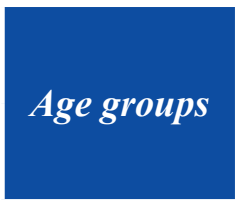

$(18-25)$

(26-35)

$(36-45)$

(46-55)

Total
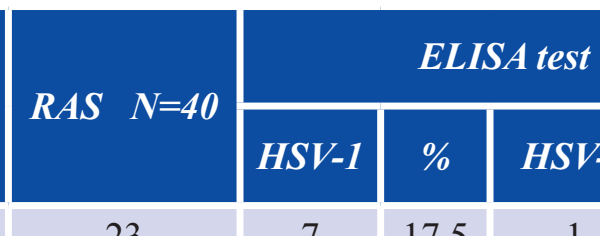

\begin{tabular}{|c|}
\hline$\%$ \\
\hline 17.5
\end{tabular}

\begin{tabular}{|c|}
\hline $\boldsymbol{H S} \boldsymbol{V}-\mathbf{2}$ \\
\hline 1 \\
\hline 2 \\
\hline 1 \\
\hline 0 \\
\hline 4 \\
\hline
\end{tabular}

\begin{tabular}{|c|}
\hline$\%$ \\
\hline 2.5 \\
\hline 5 \\
\hline 2.5 \\
\hline 0 \\
\hline 10 \\
\hline
\end{tabular}

\begin{tabular}{|c|c|}
\hline \multicolumn{2}{|c|}{ Gender } \\
\hline$M$ & $F$ \\
\hline 3 & 5 \\
\hline 2 & 4 \\
\hline 0 & 2 \\
\hline 1 & 0 \\
\hline 6 & 11 \\
\hline
\end{tabular}

\begin{tabular}{|c|c|}
$\begin{array}{c}\text { Healthy control } \\
\boldsymbol{N}=\mathbf{4 0}\end{array}$ \\
\hline$+v e$ & $-\boldsymbol{- v e}$ \\
\hline & 7 \\
\hline 2 & 5 \\
\hline 1 & 2 \\
\hline 0 & 3 \\
\hline 0 & 17 \\
\hline 3 &
\end{tabular}

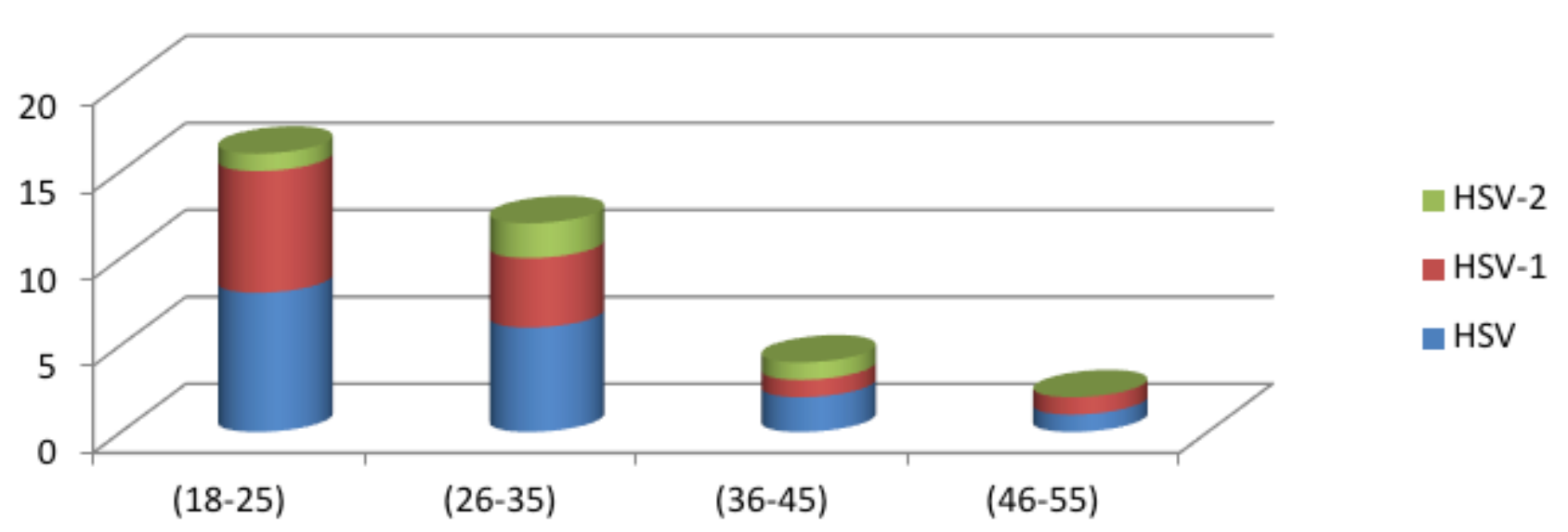

Figure (3): ELISA results of HSV in Study Age groups with Aphthus by HSV-1and HSV-2

\section{DISCUSSION}

The results included detected HSV viruses among those Iraqi patients that had diagnosed recurrent aphthous stomatitis, they confirmed with appearance with those two viruses in their saliva and this result consider most important in diagnosis and treatment, especially to adding the specific antiviral drugs in treatment plan. Also this study got the correlations between number of patients with aphthus, and age groups, gender, family history which revealed a significant relations between them. ELISA results of HSV detection and discrimination of HSV-1 and HSV-2 presence, and this brought up a new etiological result of oral infection presented with HSV-2 virus that consider a foreign causative agent in oral infection because its inhabit found in genital area, and this open 
more ideas to research about the actual reasons of transmission of this virus to colonize the oral cavity in those patients and to facilitate the treatment and prevention recurrent aphthous stomatitis in future.

1- Patients with RAS lesions: The high number of aphthus in study group were among the age group $(18-25)$ and in females were (14) more than in males (9). Also in healthy control the persons had ulcers but not aphthus were most in age group (18-25) acted (9) and this result refer to correlation between the infection with aphthus and other kinds of ulcers and these age group.

2- Age and Gender: Most RAS cases were in age group (18-25):23, in female: 14, and mostly in those with family history of infection were: 11 cases. This result confirm the predominant of this infection in younger age and mostly in females.

This result obtained because in the adult patients, "predominantly the first ulceration appeared before the age of 25 in $65-80 \%$ of patients". It was found increase in females (14) while (9) in males, and this agree with (14): "there may also be a female predisposition in affected children and a decreased prevalence has been noted in males but not females in age group (18-25) ", and over the age of 45 among Baghdad population, both genders had decrease prevalence results were (1) and this agree with (15) who found a decreasing in levels with age rise in both genders.

3- Family history: In (11) individuals with RAS were have a family history of infection with RAS in 40 patients were had a positive result of RAS with oral ulcers at there earlier age and they had severe clinical symptoms than patients with no family history of RAS. This agree with (3) when he said: "The probability of a sibling developing RAS is influenced by the parents' RAS status", and with (10) when he said "increased risk in children of two affected parents (67-90\%), as well as there being a high correlation of RAS in identical twins".

4- ELISA Results of HSV: This results proved that HSVs were founded in many cases in significant number and caused recurrent aphthus in those patients especially in female at age group (18-25), and this agree with "herpes simplex virus (HSV) has been detected in circulating mononuclear cells of RAS leisions" (19).

5- Results of HSV-1 and HSV-2: The results of ELISA to detect HSV had high significant (17): 42.5\% from (40) patients with (RAS), HSV-1: (13)32.5\%, and HSV-2: (4)10\%. This high number of RAS cases by $\mathrm{HSV}$, and presence of HSV-2 in patients saliva in this study acted important cause of prevalence of this disease in Iraq, were in age group (18-25) mostly in female especially in those with family history of infection, and this agree with ${ }^{(20)}$ when he said: "The highest prevalence of HSV-1 or HSV-2 was observed in the youngest age groups".

\section{CONCLUSIONS}

Recurrent aphthous stomatitis (RAS) is a common oral complaint and causes discomfort with significant pain and effect on life style. Most RAS cases, in this study that acted the prevalence of this disease in Iraq, were in age group (18-25) mostly in female especially in those with family history of infection. Many researches has been done to find suitable treatments for pain relief, reduce duration of disease and control of frequency and severity of ulcer outbreaks. There are many local and systemic treatment options, may help for management of aphthae in the care aids and detection of HSV-1 and HSV-2 in saliva of these patients will help in providing an important additional treatment as antiviral drugs, such as anti HSV drugs for both purposes: treatment and control of transmission among individuals, families and other shapes of populations, also the detection of HSV2 in (RAS) patients explained the mode of infection that may occurs from contact or contamination of individuals' saliva with secretions of genital organs or area that considers the habitant of HSV-2, and this could be an important point for prevention or reducing aphthous infection, transmission and even help in disease control in our country.

\section{REFERANCES:}

1. Preeti L, Magesh K, Rajkumar K, Karthik R. Recurrent aphthous stomatitis. J Oral Maxillofac Pathol. 2011;15:2526.

2. Porter SR, Leao JC. Review article: oral ulcers and its relevance to systemic disorders. Aliment Pharmacol Ther. 2015; 21:295-306.

3. Ship JA. Recurrent aphthous stomatitis. An update. Oral Surg Oral Med Oral Pathol 81, 2016: 141-7.

4. Liang MW, Neoh CY. Oral aphthosis: management gaps and recent advances. Ann Acad Med Singapore. 2012;41:46370 .

5. Baccaglini L, Lalla RV, Bruce AJ, Sartori-Valinotti JC, Latortue MC, Carrozzo M, et al. Urban legends:recurrent aphthous stomatitis. Oral Dis. 2011;17:755-70.

6. Scully C, Porter S. Oral mucosal disease:recurrent aphthous stomatitis. Br J Oral Maxillofac Surg. 2008;46:198-206.

7. Chavan M, Jain H, Diwan N, Khedkar S, Shete A, Durkar S. Recurrent aphthous stomatitis:a review. J Oral Pathol Med. 2012;41:577- 83 .

8. Magesh KT, Preeti L, Rajkumar K, Karthik R. Recurrent aphthous stomatitis. J Oral Maxillofac Pathol. 2011;15:2526. 
9. Stanley HR. Aphthous lesions. Oral Surg Oral Med Oral Pathol 33, 1972: 407-16.

10. Miller MF, Garfunkel AA, Ram C, Ship II. Inheritance patterns in recurrent aphthous ulcers: twin and pedigree data. Oral Surg Oral Med Oral Pathol 43, 1977: 886-91. [IVSL].

11. Kvam E, Gjerdet NR, Bondevik O. Traumatic ulcers and pain during orthodontic treatment. Community Dent Oral Epidemiol 15, 2007: 104-7.

12. Greenspan D, Hilton JF, Westenhouse JL. Association between oral lesions and smoking in a clinic population (Abstract no. PoB 3362). Amsterdam, Netherland, Int Conference AIDS VIII, 2002: B147.

13. Riggio MP, Lennon A, Ghodratnama F, Wray D. Lack of association between Streptococcus oralis and recurrent aphthous stomatitis. J Oral Pathol Med 29, 2000: 26-32. [IVSL].

14. Field EA, Brookes V, Tyldesley WR. Recurrent aphthous ulceration in children: a review. Int J Pediatr Dent 2, 2012 $1-10$.

15. Axéll T. A prevalence study of oral mucosal lesions in an adult Swedish population. Odontol Rev 27 (Suppl 36), 2006:1S-103S. [IVSL].
16. Jurge S, Kuffer R, Scully C, Porter SR. Mucosal disease series. Number VI. Recurrent aphthous stomatitis. Oral Dis. 2006; 12:1-21.

17. Femiano F, Lanza A, Buonaiuto C, et al. Guidelines for diagnosis and management of aphthous stomatitis. Pediatr Infect Dis J.2007; 26:728-32.

18. Neoh CY. , Liang MW, Oral aphthosis: management gaps and recent advances. Ann Acad Med Singapore. 2012;41:463-70.

19. Eglin RP, Lehner T, Subak-Sharpe JH Detection of RNA complementary to herpes-simplex virus in mononuclear cells from patients with Behcet's syndrome and recurrent oral ulcers. (1982). Lancet 2: 1356-1361.

20. A. Vahidnia1, I. Op den Buijs2, R. Roymans2, H. Bliekendaal1 and J. van de Bovenkamp. ATAL-Medical Diagnostic Centre, Amsterdam and Laboratory for Medical Microbiology, PAMM, Veldhoven, the Netherlands, Clin Microbiol Infect 2013; 19: E166-E168. 